\title{
Computer analysis of transcriptomes in glioma cells
}

\author{
S.S. Kovalev ${ }^{*}$, N.V. Gubanova², A.V. Tsukanov², A.O. Bragin ${ }^{2}$, Y.L. Orlov²,3 \\ ${ }^{1}$ Novosibirsk State Medical University, Novosibirsk, Russia \\ ${ }^{2}$ Institute of Cytology and Genetics SB RAS, Novosibirsk, Russia \\ ${ }^{3}$ Novosibirsk State University, Novosibirsk, Russia \\ *e-mail: sergey.kovalev.1994@list.ru
}

Key words: cancer, transcriptomics, database, gene expression regulation, splicing, glioma

Motivation and Aim: Glioma is the most common malignant tumor in the central nervous system. Here we consider problem of detection of genes responsible for glioma progression in primary cell cultures. The identification of sensitive and specific biological markers that would help identify patients at a higher or lower risk of death from glioma is of crucial importance [1]. We revealed set of differently expressed gene in normal brain and glioma cell cultures and analyzed alternative splicing variants.

Methods and Algorithms: The primary cell culture samples from normal brain and secondary glioblastoma were processed for RNA extraction. This was followed by RNA-sequencing, data processing and filtration of reads. The algorithm of the pipeline consists of several stages: 1) pre-processing of the reads; 2) mapping reads to the reference genome; 3 ) identification of differential expression and cases of alternative splicing; 4) annotation of obtained results. For assessment of gene expression level and finding differently expressed genes we used Cufflinks. Set of computer tools were used for sequencing data processing and alternative splicing analysis [2].

Results: We found set of hormone transporter genes overexpressed in the glioblastoma cell culture. SLCO1C1 mediates the Na+-independent high affinity transport of organic anions such as the thyroid hormones thyroxine. Multiple alternative splicing transcripts have been identified as progression markers. We found set of differentially alternatively spliced transcripts. 73 of the differentially expressed genes were found in OMIM as related to glioma (of 193 loci) including GLI1, GLI3 (GLI-Kruppel family member 3); GLM4 (GLIOMASUSCEPTIBILITY4), GLTSCR1 (Glioma tumor suppressor candidate region gene 1) and others. We have developed database of genes with alternative splicing in glioma including APP, CASC4 and TP53.

Conclusion: The RNA-seq analysis of the cells cultures of normal brain and glioma confirmed association of these genes with tumor progression. The results provide an experimental basis for the observation that hypothyroidism induction by administration of propylthiouracil is associated with improved survival in glioblastoma patients. We work on integration of data on glioma genes using available data sources developing own database prototype.

Acknowledgements: The research has been supported by ICG SB RAS budget project (0324-2018-0021). Computing at Siberian Supercomputer center SB RAS (SSCC) was done in frames of the budget project 0324-2018-0017.

\section{References}

1. Liu M. et al. (2017) The identification of key genes and pathways in glioma by bioinformatics analysis. Journal Immunology Research. Article ID 1278081.

2. Babenko V.N. et al. (2017) Computer analysis of glioma transcriptome profiling: alternative splicing events. J Integr Bioinform. 14(3). pii: /j/jib.2017.14.issue-3/jib-2017-0022/jib-2017-0022.xml. 